\title{
A 3D semi-anthropomorphic photoacoustic breast phantom
}

Maura Dantuma, Rianne C. van Dommelen, Srirang Manohar

Maura Dantuma, Rianne C. van Dommelen, Srirang Manohar, "A 3D semianthropomorphic photoacoustic breast phantom," Proc. SPIE 10878, Photons Plus Ultrasound: Imaging and Sensing 2019, 108781P (27 February 2019); doi: $10.1117 / 12.2506873$

SPIE. Event: SPIE BiOS, 2019, San Francisco, California, United States 


\title{
A 3D semi-anthropomorphic photoacoustic breast phantom
}

\author{
Maura Dantuma ${ }^{\mathrm{a}}$, Rianne C. van Dommelen ${ }^{\mathrm{a}}$, and Srirang Manohar ${ }^{\mathrm{a}}$ \\ ${ }^{a}$ Biomedical Photonic Imaging group, Technical Medical Centre, University of Twente, 7522 \\ NB Enschede, The Netherlands
}

\begin{abstract}
Photoacoustic tomographic breast imaging systems progressively move into the clinics for in-vivo studies. Next to tumor detection, studies also focus on extracting information about the tumor by performing multi-wavelength photoacoustics for quantitative oxygen saturation estimations. Until now, it has been difficult to compare the results from different systems due to the wide variability in system characteristics and image reconstruction algorithms. In order to do inter-system comparisons in photoacoustic breast imaging, and to validate oxygen saturation estimations, a standardized but realistic measurement object is required. In this study, we present the first 3D semi-anthropomorphic photoacoustic breast phantom and demonstrate its features both in ultrasound imaging as in photoacoustic tomography.
\end{abstract}

Keywords: photoacoustic imaging, optoacoustic imaging, breast phantom, 3D printing, polyvinyl chloride plastisol (PVCP)

\section{INTRODUCTION}

The application of photoacoustic (PA) imaging in breast cancer screening and diagnosis is still in its infancy, but more and more applications are being found. ${ }^{1-3}$ Blood vessels inside the breast can be visualized, but multiwavelength PA imaging has shown to be able to provide quantitative oxygen saturation estimations. ${ }^{4}$ Several devices are being developed and/or optimized for clinical trials at the moment. The differing system geometries, components and algorithms these devices possess all influence the imaging performance, which makes it difficult to do valid inter-system comparison and thereby to finally and at optimized system configurations for certain applications.

To be able to do these inter-system comparisons a phantom fabrication protocol is required, which opens up the possibility to make identical measurement objects in all the labs. When using a semi-realistic PA breast phantom, it can be utilized as well e.g. to compare and test quantitative PA algorithms. Research has been performed into suitable tissue mimicking materials (TMM) for PA imaging and elemental phantoms have been developed, ${ }^{5-12}$ but no protocols for 3D PA breast phantoms have been reported.

The introduction of multi-material 3D printing simplifies the development of complex phantoms for optical imaging since the phantoms can be built up layer by layer allowing complex structures. Examples can be found for breast x-ray imaging ${ }^{13}$ and fluorescent brain imaging. ${ }^{14}$ However, due to limited available 3D print materials, it is not possible yet to print PA phantoms with both optical and acoustic properties matching the body's soft tissues. However, the utilization of 3D print techniques for mold making enables us to build a novel 3D semi-anthropomorphic PA breast phantom, containing the breast's four most prevalent tissue types being skin, fat, fibroglandular and blood.

Vogt et al. ${ }^{5,15}$ show that polyvinyl chloride plastisol (PVCP) combined with scattering and absorbing additives, can be used to mimic the breasts most prevalent tissues both acoustically as optically and that the material has a good longevity, making it a promising PA phantom material. In this study we build further upon this work and present a protocol for phantom fabrication together with the measured acoustic and optical properties of the four TMM. We end with a demonstration of the phantom's features in ultrasound imaging and PA tomography.

Correspondence address: m.dantuma@utwente.nl

Photons Plus Ultrasound: Imaging and Sensing 2019, edited by Alexander A. Oraevsky, Lihong V. Wang, Proc. of SPIE Vol. 10878, 108781P - (C) 2019 SPIE · CCC code: 1605-7422/19/\$18 - doi: 10.1117/12.2506873 


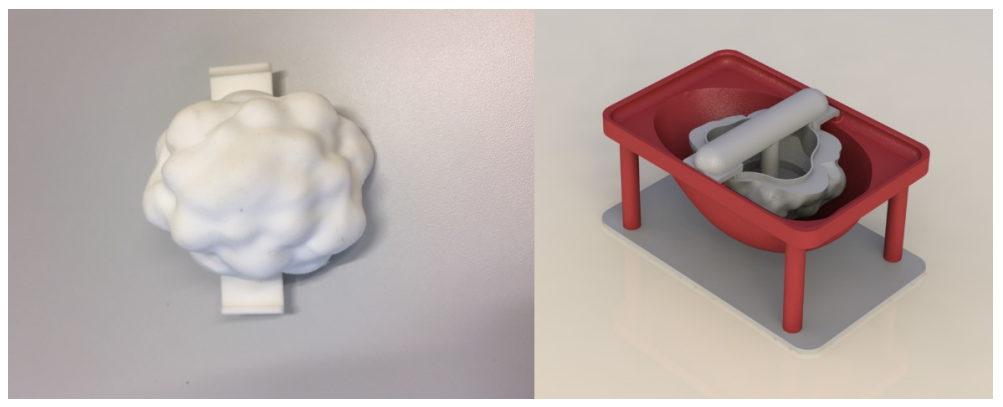

Figure 1: Digital model of the mold. Red = outer mold, shaped like the outer contours of a breast. White $=$ inner mold representing the fibroglandular tissue.

\section{MATERIALS AND METHODS}

\subsection{Phantom morphology and fabrication protocol}

Breasts differ a lot in shape and size, but all contain the four tissue types mentioned before. The ratio of fibroglandular versus fat tissue defines the breast density and differs from individual to individual. The morphology of our phantom is based on a 3D numerical phantom of a heterogeneously dense breast segmented from MRI by Y. Lou et al. ${ }^{16}$ Due to the high complexity of the fibroglandular structure in the numerical phantom making it impossible to print molds for this shape, it was decided to simplify the shape of this structure. Fibroglandular tissue consists of multiple lobes, with some fat areas interwoven. This lobular shape is assimilated in the phantom by making use of a 3D cauliflower model.

These models were converted into nylon molds (see Figure 1) by making use of 3D printing. The phantom can be produced in a few steps once all the TMM mixtures are prepared (see section 2.2 for the protocols). First, the skin is produced in the outside mold by applying a 1-2 mm thick silicone rubber layer to the surface of the outer mold. Meanwhile, tumors and blood vessels can be created by cutting preferred shapes from a slab of blood TMM. Some of these structures can then be placed in the outer mold. Subsequently, the fat TMM can be poured in between the skin layer and the inner mold. After solidification, the outer mold can be removed and remaining tumor/blood vessels can be positioned inside the opening. Fill the hole with fibroglandular TMM, and finally top the entire phantom off with an additional PVCP layer for extra stability and attachment to a holder (Figure 3c).

\subsection{Tissue mimicking materials}

Vogt et al. characterized the acoustic properties of several PVC-plasticizer combinations, and tuned acoustic and optical properties of the materials with the addition of beads and coloring paste. ${ }^{5,15}$ We have used their findings in developing the fat, fibroglandular and blood/tumor mimicking materials:

PVC resin (10 m/m\%) (Geon 121A, Mexichem, Mexico) was mixed with benzyl butyl phthalate (BBP) and bis(2-ethylhexyl) adipate (DEHA), $41.6 \mathrm{v} / \mathrm{v} \%$ and $57.4 \mathrm{v} / \mathrm{v} \%$ respectively, to mimic fat tissue. $6 \mathrm{mg} / \mathrm{cm}^{3}$ glass beads (Sigma Aldrich, $\leq 106 \mu \mathrm{m}$ ) and and $1 \mathrm{mg} / \mathrm{ml} \mathrm{TiO} 2$ beads were added to tune the acoustic and optical scattering respectively. Fibroglandular TMM was obtained by using $99 \mathrm{v} / \mathrm{v} \%$ BBP, $30 \mathrm{mg} / \mathrm{cm}^{3}$ glass beads and $1.6 \mathrm{mg} / \mathrm{cm}^{3} \mathrm{TiO}_{2}$ beads, resulting in higher acoustic and optical scattering. Tumor/blood TMM was made with the same protocol, however, glass beads were omitted and $0.2 \mathrm{v} / \mathrm{v} \%$ Black plastic coloring (Lure Parts) was added. The skin TMM was made from silicon rubber (polyestershoppen.nl) as a layer between the mold and the fat to easily release the phantom from the mold. Silicon component A $(90 \mathrm{~m} / \mathrm{m} \%)$ was mixed with silicon component B $(9 \mathrm{~m} / \mathrm{m} \%)$. Silicon thickener $(0.5 \mathrm{~m} / \mathrm{m} \%)$ was added together with orange $(0.08 \mathrm{~m} / \mathrm{m} \%)$ and white $(0.54 \mathrm{~m} / \mathrm{m} \%)$ pigment paste.

\subsection{Phantom characterization}

The acoustic material properties were characterized with a modified insertion method. ${ }^{17}$ Figure 2 shows the used set-up which was placed in water. Transmission measurements were performed through two different 


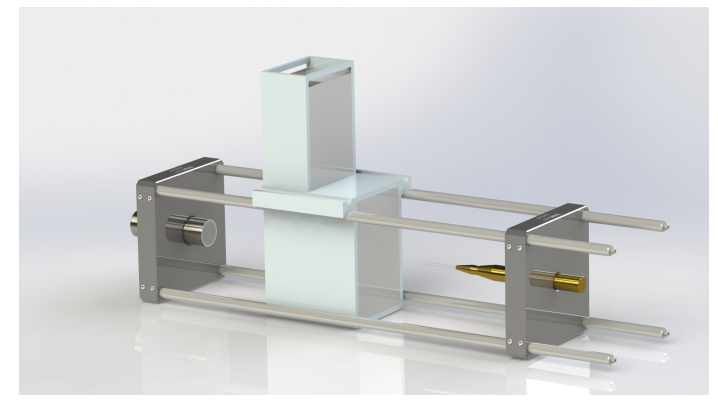

Figure 2: Set-up for the modified insertion method to measure the materials speed of sound and acoustic attenuation. Ultrasound propagates though a thinner piece of material when flipping the test block upside down. Components from left to right: Transducer, TMM in holder, hydrophone needle.

thicknesses of the material, to minimize inaccuracies coming from reflections at the interfaces. A $1 \mathrm{MHz}$ single element unfocused immersion transducer (Panametrics) was used together with a hydrophone needle (Precision Acoustics) connected to pre-amplifier. Test blocks of the TMM were made, and the speed of sound (SoS) and acoustic attenuation (AA) were extracted by looking at the time of arrival and amplitude of the transmitted waves. The density of the material was measured to calculate the acoustic impedance. Optical absorption and scattering were measured with the inverse adding-doubling (IAD) method. ${ }^{18}$ A spectrophotometer with integrating sphere (Shimadzu UV2600, Kyoto, Japan) measured both transmittance as reflection on thin PVCP slabs.

\subsection{Imaging}

The phantom was imaged with a $6 \mathrm{MHz}$ linear probe (Alpinion, E-Cube12R, Korea, L3-12) to investigate its appearance in ultrasound imaging. Additionally, it was imaged with the Twente Photoacoustic Mammoscope 2 (PAM2). ${ }^{19,20}$ The phantom was illuminated with $755 \mathrm{~nm}$ pulses fired by an Alexandrite laser with a duration of $60 \mathrm{~ns}$. Twelve arc-shaped ultrasound detector arms, each containing 32 elements with a $1 \mathrm{MHz}$ center frequency, rotated 60 degrees around the phantom in 45 steps. Data was acquired for all detector positions. Images were reconstructed with a filtered back projection algorithm.

\section{RESULTS}

The phantom was made according to the protocol. Figure $3 \mathrm{~b}$ and $\mathrm{c}$ show the phantom under construction together with the final result.

\subsection{Phantom characterization}

Table 1 summarizes the measured properties and compares them to average literature values. The optical measurements are not reported here due to inaccuracies in the values estimated. This is most likely due to a bug in the IAD algorithm implemented. This has been resolved, but values have not been corrected at the time of going to press. They will be reported in a later publication.

\subsection{Imaging}

Figure 4 shows the images acquired with US and PA imaging. All four tissue types can be observed in the US image. The skin shows as a bright region in the top part of the image. The fibroglandular layer contains more scatterers than the fat layer, which makes it brighter and enables the visibility of the fibroglandular-fat interface. The tumor does not contain any scatterers which makes it appear black.

Figure 5 shows a color-coded local maximum intensity projections of the PA measurement. The optical absorbing structures show up well in the images. The tumors do not show up as a solid structures, which may be explained by the fact that large objects generate low frequency signals which lie outside of the detectors' 
bandwidth. The edges of the tumors are visualized, because of the higher frequency content they generate. A haze is visible in the top of the image, which might be caused by the skin or could be an artifact coming from the metal plate of the mounting system.

Table 1: Results of the acoustic characterization measurements. Acoustic attenuation was measured at $1 \mathrm{MHz}$. Literature values are in brackets.

\begin{tabular}{lccc} 
Tissue & SoS $[\mathbf{m} / \mathbf{s}]$ & AA $[\mathbf{d B} / \mathbf{c m}]$ & $\mathbf{Z}[$ MRayls $]$ \\
\hline Skin & - & - & - \\
& $(1607 \pm 32)$ & $(1.22 \pm 0.83)$ & $(1.85)$ \\
Fat & $1477 \pm 1$ & $0.42 \pm 0.005$ & $1.55 \pm 0.06$ \\
& $(1440 \pm 20)$ & $(1-1.8)$ & $(1.31)$ \\
Fibroglandular & $1511 \pm 3$ & $0.55 \pm 0.008$ & $1.79 \pm 0.05$ \\
& $(1524 \pm 27)$ & $(2-2.7)$ & $(1.59)$ \\
Blood/tumor & $1511 \pm 3$ & $0.9 \pm 0.005$ & $1.82 \pm 0.05$ \\
& $(1582 \pm 5)$ & $(0.15 \pm 0.01)$ & $(1.67)$
\end{tabular}

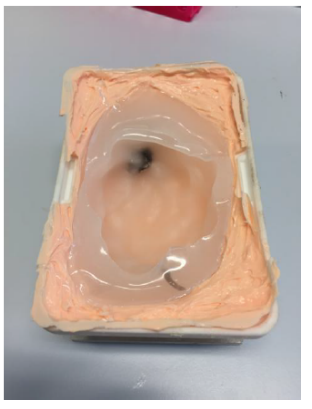

(a)

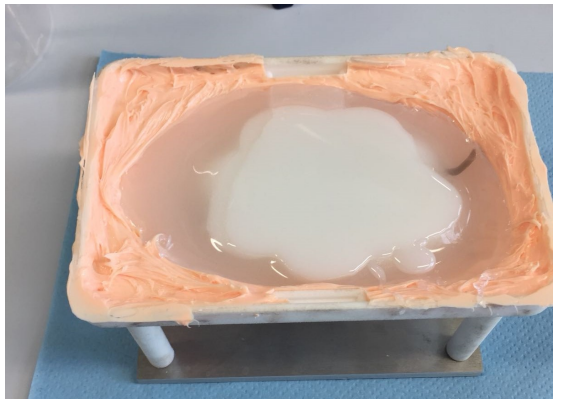

(b)

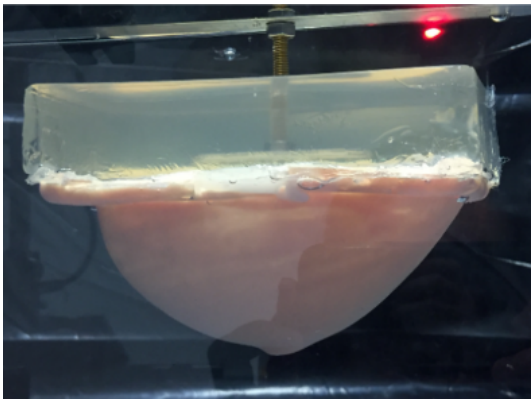

(c)

Figure 3: (a,b) Phantom during the fabrication process. The different TMM are visible. (c) Completed phantom immersed in a water tank for acoustical measurements.
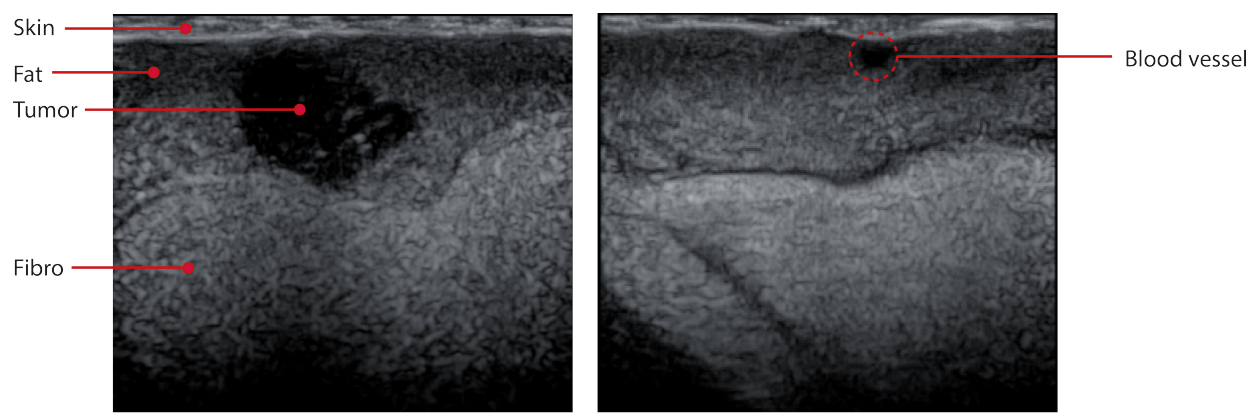

Figure 4: B-mode ultrasound images obtained with the Alpinion system. The different tissue layers can be observed. 
(b)

(a)

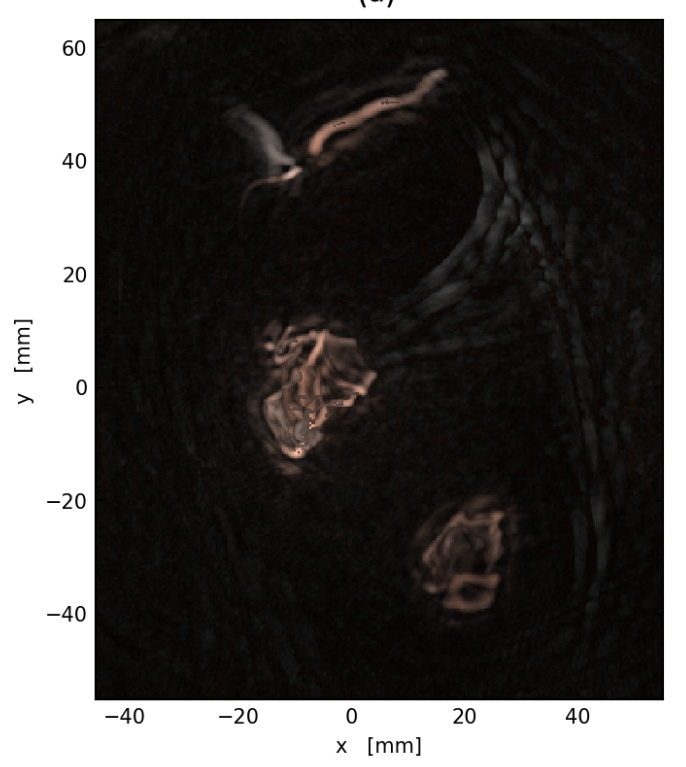

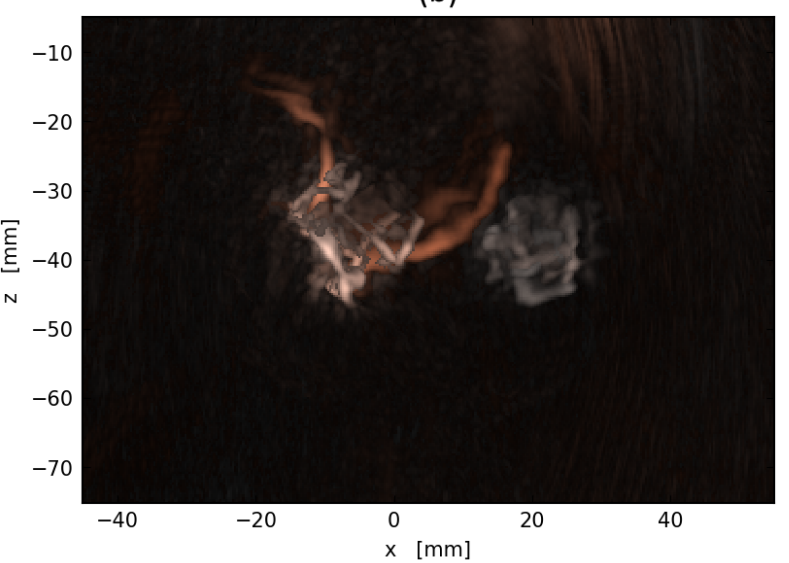

(c)

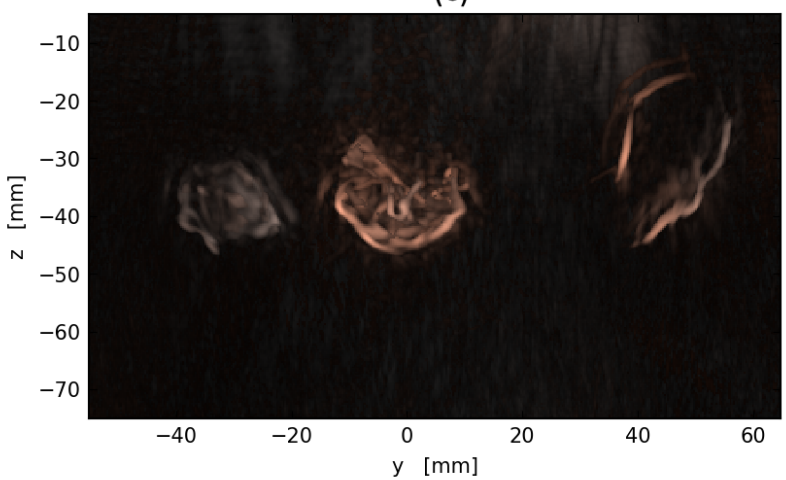

Figure 5: Color-coded local maximum intensity projections along three planes of the 3D PA data set. bright $=$ superficial, dark $=$ deep.

\section{CONCLUSION AND OUTLOOK}

We report on the first 3D PA breast phantom. The materials are based on PVCP compositions and the complexity of the breast is mimicked by using novel 3D printed molds. We have described the fabrication steps. The four TMM have been comprehensively characterized acoustically and optically. The phantom's appearance in US and PA has been demonstrated. All four tissue types can be observed in US imaging. When looking at the PA performance, a clear difference with PA patient measurements is the absence of the nipple in the image. Therefore, in future phantoms the absorption in the nipple area will be increased. Also more realistic blood vessels and tumor structures will be embedded in later versions of this phantom. The acoustic attenuation and the optical properties require optimization, but methods to do this are known.

\section{ACKNOWLEDGMENTS}

The authors thank Sjoukje Schoustra and Tim op 't Root for performing the PA measurement and reconstructing the images. They also like to thank Vincent Groenhuis and Françoise Siepel from the Robotics and Mechatronics group for allowing the usage of their 3D printers, and for thinking along with the project. This work is part of the PAMMOTH project, and is made possible by the Horizon 2020's research and innovation program, H2020 ICT 2016-2017, under grant agreement No 732411 which is an initiative of the Photonics Public Private Partnership. 


\section{REFERENCES}

[1] Oraevsky, A., Su, R., Nguyen, H., Moore, J., Lou, Y., Bhadra, S., Forte, L., Anastasio, M., and Yang, W., "Full-view 3d imaging system for functional and anatomical screening of the breast," in [Photons Plus Ultrasound: Imaging and Sensing 2018], 10494, 104942Y, International Society for Optics and Photonics (2018).

[2] Lin, L., Hu, P., Shi, J., Appleton, C. M., Maslov, K., Li, L., Zhang, R., and Wang, L. V., "Single-breath-hold photoacoustic computed tomography of the breast," Nature Communications 9(1), 2352 (2018).

[3] Heijblom, M., Piras, D., van den Engh, F. M., van der Schaaf, M., Klaase, J. M., Steenbergen, W., and Manohar, S., "The state of the art in breast imaging using the twente photoacoustic mammoscope: results from 31 measurements on malignancies," European Radiology 26(11), 3874-3887 (2016).

[4] Li, X., Heldermon, C. D., Yao, L., Xi, L., and Jiang, H., "High resolution functional photoacoustic tomography of breast cancer," Medical Physics 42(9), 5321-5328 (2015).

[5] Jia, C., Vogt, W. C., Wear, K. A., Pfefer, T. J., and Garra, B. S., "Two-layer heterogeneous breast phantom for photoacoustic imaging," Journal of Biomedical Optics 22(10), 106011 (2017).

[6] Cook, J. R., Bouchard, R. R., and Emelianov, S. Y., "Tissue-mimicking phantoms for photoacoustic and ultrasonic imaging," Biomedical Optics Express 2(11), 3193-3206 (2011).

[7] Fonseca, M., Zeqiri, B., Beard, P., and Cox, B., "Characterisation of a phantom for multiwavelength quantitative photoacoustic imaging," Physics in Medicine 83 Biology 61(13), 4950 (2016).

[8] Bohndiek, S. E., Bodapati, S., Van De Sompel, D., Kothapalli, S.-R., and Gambhir, S. S., "Development and application of stable phantoms for the evaluation of photoacoustic imaging instruments," PloS One 8(9), e75533 (2013).

[9] Spirou, G. M., Oraevsky, A. A., Vitkin, I. A., and Whelan, W. M., "Optical and acoustic properties at $1064 \mathrm{~nm}$ of polyvinyl chloride-plastisol for use as a tissue phantom in biomedical optoacoustics," Physics in Medicine \& Biology 50(14), N141 (2005).

[10] Kharine, A., Manohar, S., Seeton, R., Kolkman, R. G., Bolt, R. A., Steenbergen, W., and de Mul, F. F., "Poly (vinyl alcohol) gels for use as tissue phantoms in photoacoustic mammography," Physics in Medicine E) Biology 48(3), 357 (2003).

[11] Manohar, S., Kharine, A., Steenbergen, W., and van Leeuwen, T. G., "Three-dimensional photoacoustic imaging of breast tissue phantoms," in [ALT'03 International Conference on Advanced Laser Technologies: Biomedical Optics], 5486, 247-252, International Society for Optics and Photonics (2004).

[12] Xia, W., Piras, D., Heijblom, M., Steenbergen, W., Van Leeuwen, T. G., and Manohar, S., "Poly (vinyl alcohol) gels as photoacoustic breast phantoms revisited," Journal of Biomedical Optics 16(7), 075002 (2011).

[13] Carton, A.-K., Bakic, P., Ullberg, C., Derand, H., and Maidment, A. D., "Development of a physical 3d anthropomorphic breast phantom," Medical Physics 38(2), 891-896 (2011).

[14] Liu, Y., Ghassemi, P., Depkon, A., Iacono, M. I., Lin, J., Mendoza, G., Wang, J., Tang, Q., Chen, Y., and Pfefer, T. J., "Biomimetic 3d-printed neurovascular phantoms for near-infrared fluorescence imaging," Biomed. Opt. Express 9, 2810-2824 (Jun 2018).

[15] Vogt, W. C., Jia, C., Wear, K. A., Garra, B. S., and Pfefer, T. J., "Biologically relevant photoacoustic imaging phantoms with tunable optical and acoustic properties," Journal of Biomedical Optics 21(10), 101405 (2016).

[16] Lou, Y., Zhou, W., Matthews, T. P., Appleton, C. M., and Anastasio, M. A., "Generation of anatomically realistic numerical phantoms for photoacoustic and ultrasonic breast imaging," Journal of Biomedical Optics 22(4), 041015 (2017).

[17] Xia, W., Piras, D., van Hespen, J. C., Steenbergen, W., and Manohar, S., "A new acoustic lens material for large area detectors in photoacoustic breast tomography," Photoacoustics 1(2), 9-18 (2013).

[18] Prahl, S. A., van Gemert, M. J., and Welch, A. J., "Determining the optical properties of turbid media by using the adding-doubling method," Applied Optics 32(4), 559-568 (1993).

[19] Schoustra, S. M., Piras, D., Huijink, R., op t Root, Tim, J. P. M., Alink, L., Muller Kobold, W. F., Steenbergen, W., and Manohar, S., "The twente photoacoustic mammoscope 2: system overview and 3d vascular network images in healthy breasts," in preparation (2019). 
[20] Schoustra, S. M., Huijink, R., Alink, L., op t Root, Tim, J. P. M., Sprnken, D., Piras, D., Muller Kobold, W. F., Klazen, C. A. H., van der Schaaf, M. C., van den Engh, F. M., Steenbergen, W., and Manohar, S., "The twente photoacoustic mammoscope 2: instrument optimization and 3d vascular network visualization," Proceedings of SPIE, Paper number 10878-37, to be published, SPIE (2019). 